\title{
STUDY OF PROPERTIES OF SEVERAL KINDS OF HYPERIDEALS IN ORDERED TERNARY SEMIHYPERGROUPS
}

\author{
Thawhat Changphas ${ }^{1}$, And Bijan Davvaz ${ }^{2}$ \\ ${ }^{1}$ Department of Mathematics, Faculty of Science, Khon Kaen University, \\ Khon Kaen 40002, Thailand, thacha@kku.ac.th \\ ${ }^{2}$ Department of Mathematics, Yazd University, Yazd, Iran, \\ davvaz@yazd.ac.ir
}

\begin{abstract}
A non-empty subset $S$ together with an associative function $f$ from $S \times$ $S \times S$ into the family of all non-empty subsets of $S$ is called a ternary semihypergroup. In this paper, we consider a semihypergroup $(S, f)$ besides a binary relation $\leq$, where $\leq$ is a partial order relation on $S$ such that satisfies the monotone condition. This structure is called an ordered ternary semihypergroup. We introduce and investigate the notions of bi-hyperideal and quasi-hyperideal in ordered ternary semihyperroups. In particular, we prove that an ordered ternary semihypergroup is left and right simple if and only if it does not contain proper bi-hyperideals.

Key words and Phrases: Algebraic hyperstructure, ordered ternary semihypergrouip, ternary subsemihypergroup, (bi-, quasi-) hyperideal, prime hyperideal, quasi-simple.
\end{abstract}

\section{INTRODUCTION}

The idea of investigations of $n$-ary algebras, i.e., sets with one $n$-ary operation, seems to be going back to Kasner's lecture, but the first paper about the subject was written by Dörnte [9]. The notion of ternary algebraic system was first introduced by Lehmer [22] in 1932. He investigated certain ternary algebraic systems called triplexes which turn out to be commutative ternary groups. The notion of ternary semigroups was introduced by Banach, see [24]. He showed by an example that a ternary semigroup does not necessarily reduce to an ordinary semigroup. Los [24] studied some properties of ternary semigroup and proved that every ternary semigroup can be embedded in a semigroup. These two algebraic structures were

2020 Mathematics Subject Classification: 20N20, 06F05.

Received: 18-10-2020, accepted: 20-07-2021. 
further studied by different authors. The ideal theory in ternary semigroups was studied by Sioson [26]. He also introduced the notion of regular ternary semigroups and characterized them by using the notion of quasi-ideals. Recently, Santiago and Bala [25] developed the theory of ternary semigroups. Applications of ideals to the divisibility theory in ternary and $n$-ary semigroups and rings one can find in [10].

The concept of algebraic hyperstructures was introduced in 1934 by Marty [17] and has been studied in the following decades and nowadays by many mathematicians. Semihypergroups are studied by many authors, for example, Bonansinga and Corsini [2], Davvaz [4, 5], De Salvo et al. [8], Freni [11], Hila et al. [14, 15], Leoreanu [22], and many others. The concept of ordering hypergroups investigated by Chvalina [3] as a special class of hypergroups and studied by him and many others. In [12], Heidari and Davvaz studied a semihypergroup $(S, \circ)$ besides a binary relation $\leq$, where $\leq$ is a partial order relation such that satisfies the monotone condition.

In [7], Davvaz and Vougiouklis introduced the concept of $n$-ary hypergroups as a generalization of hypergroups in the sense of Marty. Leoreanu-Fotea and Davvaz in [23] introduced and studied the notion of a partial $n$-ary hypergroupoid, associated with a binary relation. In [1], some representation techniques for chromatic $n$-ary polygroups are presented and it is proved that the direct product of two $n$-ary color schemes and the class of $n$-ary groups by a full conjugation relation on $n$-ary polygroups is chromatic. In [18], Mirvakili and Davvaz determined two families $P(H)$ and $P_{\sigma}(H)$ of subsets of an $n$-ary hypergroup $H$ such that two geometric spaces $(H, P(H))$ and $\left(H, P_{\sigma}(H)\right)$ are strongly transitive. Also, see [19]. Ternary semihypergroups are algebraic structures with one associative hyperoperation. A ternary semihypergroup is a particular case of an $n$-ary semihypergroup for $n=3$. Ternary semihypergroups are studied by Davvaz and Leoreanu-Fotea in [6]. In [20] Naka and Hila gave some properties of left (right) and lateral hyperideals in ternary semihypergroups. They introduced the notion of left simple, lateral simple, left (0-)simple and lateral 0-simple ternary semihypergroups and characterized the minimality and maximality of left (right) and lateral hyperideals in ternary semihypergroups. Also, see [13].

Now, in this paper we study properties of several kinds of hyperideals in ordered ternary semihypergroups. The paper is structured as follows. After an introduction, in Section 2 we present some basic notions and examples of ordered ternary semihypergroups. In Section 3, we introduce the notion of bi-hyperideals of an ordered ternary semihypergroup and we give some results in this respect. In particular, we prove that an ordered ternary semihypergroup is left and right simple if and only if it does not contain proper bi-hyperideals. Finally, in the last section, we define the notion of quasi-hyperideals of an ordered ternary semihypergroup and study some of their properties.

\section{BASIC DEFINITIONS AND EXAMPLES}

Let $H$ be a non-empty set. Then $H$ is called a ternary semihypergroup [6] if there exists a ternary hyperoperation $f: H \times H \times H \rightarrow \mathcal{P}^{*}(H)$ written as 
$\left(x_{1}, x_{2}, x_{3}\right) \mapsto f\left(x_{1}, x_{2}, x_{3}\right)$, where $\mathcal{P}^{*}(H)$ is the family of all non-empty subsets of $H$, such that $f$ is an associative hyperoperation, i.e.,

$$
f\left(f\left(x_{1}, x_{2}, x_{3}\right), x_{4}, x_{5}\right)=f\left(x_{1}, f\left(x_{2}, x_{3}, x_{4}\right), x_{5}\right)=f\left(x_{1}, x_{2}, f\left(x_{3}, x_{4}, x_{5}\right)\right)
$$

for all $x_{1}, x_{2}, x_{3}, x_{4}, x_{5}$ in $H$. If $A_{1}, A_{2}$ and $A_{3}$ are non-empty subsets of a ternary semihypergroup $(H, f)$, we write

$$
f\left(A_{1}, A_{2}, A_{3}\right)=\bigcup_{a_{1} \in A_{1}, a_{2} \in A_{2}, a_{3} \in A_{3}} f\left(a_{1}, a_{2}, a_{3}\right) .
$$

An ordered ternary semihypergroup $(H, f, \leq)$ is a ternary semihypergroup $(H, f)$ together with a partial order $\leq$ that is compatible with the ternary hyperoperation, i.e., for any $x, y, a_{1}, a_{2}$ in $H$,

$$
x \leq y \Rightarrow\left\{\begin{array}{l}
f\left(x, a_{1}, a_{2}\right) \leq f\left(y, a_{1}, a_{2}\right) \\
f\left(a_{1}, x, a_{2}\right) \leq f\left(a_{1}, y, a_{2}\right) \\
f\left(a_{1}, a_{2}, x\right) \leq f\left(a_{1}, a_{2}, y\right)
\end{array}\right.
$$

Here $f\left(x, a_{1}, a_{2}\right) \leq f\left(y, a_{1}, a_{2}\right)$ means for any $u \in f\left(x, a_{1}, a_{2}\right)$ there exists $v \in$ $f\left(y, a_{1}, a_{2}\right)$ such that $u \leq v$. The cases $f\left(a_{1}, x, a_{2}\right) \leq f\left(a_{1}, y, a_{2}\right)$ and $f\left(a_{1}, a_{2}, x\right) \leq$ $f\left(a_{1}, a_{2}, y\right)$ are defined similarly.

Example 2.1. Let $H$ be a non-empty set and $\leq$ a partial order relation on $H$. For every $x, y, z$ in $H$, we define

$$
f(x, y, z)=\{x, y, z\} .
$$

Then $(H, f, \leq)$ is an ordered ternary semihypergroup.

Example 2.2. Let $(S, \cdot, \leq)$ is an ordered semigroup. We define a ternary hyperoperation on $S$ as follows: for every $x, y, z$ in $S$,

$$
f(x, y, z)=\langle x, y, z\rangle
$$

where $\langle x, y, z\rangle$ is the ideal of $S$ generated by $\{x, y, z\}$. Then $(S, f, \leq)$ is an ordered ternary semihypergroup.

Example 2.3. Let $H$ be a non-empty set and $\leq$ a totally order relation on $H$. We define a ternary hyperoperation on $H$ as follows: for every $x, y, z$ in $H$,

$$
f(x, y, z)=\{a \in H \mid x \vee y \vee z \leq a\} .
$$

Then $(H, f, \leq)$ is an ordered ternary semihypergroup.

Remark 2.4. Note that if for all $x, y, z \in S, f(x, y, z)$ is singleton set, then $(S, f, \leq)$ is an ordered ternary semigroup [16]. So, the definition of an ordered semihypergroup is a generalization of the definition of an ordered ternary semigroup, too.

Example 2.5. Let $S=\{i, 0,-i\}$ and consider the ternary operation $f$ on $S$ as the ordinary ternary multiplication of complex numbers on $S$. If we define the following order on $S$ :

$$
\leq=\{(i, i),(0,0),(-i,-i),(i, 0),(-i, 0)\}
$$

then, $(S, f, \leq)$ is an ordered semigroup as well as an ordered semihypergroup. 
A non-empty subset $A$ of an ordered ternary semihypergroup $(H, f, \leq)$ is called a ternary subsemihypergroup if $f(A, A, A) \subseteq A$.

Definition 2.6. A non-empty subset $A$ of an ordered ternary semihypergroup $(H, f, \leq)$ is called a left (respectively, middle, right) hyperideal if it satisfies the following conditions:

(i) $f(H, H, A) \subseteq A$ (respectively, $f(H, A, H) \subseteq A, f(A, H, H) \subseteq A$ );

(ii) if $x \in A$ and $y \in H$ such that $y \leq x$, then $y \in A$.

If $A$ is both a left and a right hyperideal of $H$, then it is called a two-sided hyperideal of $H$. If $A$ is a middle and a two-sided hyperideal of $H$, then it is called a hyperideal of $H$.

Example 2.7. Let $H=\{1,2,3\}$. We define a ternary hyperoperation $f$ and a partial order as follows:

\begin{tabular}{|l|l|l|}
\hline$(1,1,1) \mapsto 1$ & $(2,1,1) \mapsto 1$ & $(3,1,1) \mapsto 1$ \\
$(1,1,2) \mapsto\{1,2\}$ & $(2,1,2) \mapsto\{1,2\}$ & $(3,1,2) \mapsto\{1,2\}$ \\
$(1,1,3) \mapsto\{1,3\}$ & $(2,1,3) \mapsto\{1,3\}$ & $(3,1,3) \mapsto\{1,3\}$ \\
$(1,2,1) \mapsto 1$ & $(2,2,1) \mapsto 1$ & $(3,2,1) \mapsto 1$ \\
$(1,2,2) \mapsto\{1,2\}$ & $(2,2,2) \mapsto 2$ & $(3,2,2) \mapsto\{1,2\}$ \\
$(1,2,3) \mapsto\{1,3\}$ & $(2,2,3) \mapsto\{1,3\}$ & $(3,2,3) \mapsto\{1,3\}$ \\
$(1,3,1) \mapsto 1$ & $(2,3,1) \mapsto 1$ & $(3,3,1) \mapsto 1$ \\
$(1,3,2) \mapsto\{1,2\}$ & $(2,3,2) \mapsto\{1,2\}$ & $(3,3,2) \mapsto\{1,2\}$ \\
$(1,3,3) \mapsto\{1,3\}$ & $(2,3,3) \mapsto\{1,3\}$ & $(3,3,3) \mapsto\{1,3\}$ \\
\hline \multicolumn{3}{|c|}{$\leq=\{(1,1),(2,2),(3,3),(2,1),(3,1)\}}$.
\end{tabular}

Then $(H, f, \leq)$ is an ordered ternary semihypergroup with the covering relation and the figure of $H$ as follows:

$$
<=\{(2,1),(3,1)\}
$$

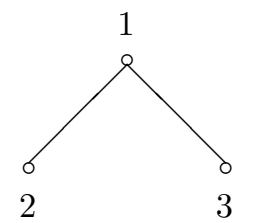

We have $A=\{1,2\}, B=\{1,3\}$ and $C=\{1\}$ are left hyperideals of $H$.

Let $A$ be a non-empty subset of an ordered ternary semihypergroup $(H, f, \leq)$. Define

$$
(A]=\{x \in H \mid x \leq a \text { for some } a \in A\} .
$$

Note that the condition (ii) in Definition 2.6 is equivalent to $A=(A]$. If $A_{1}, A_{2}$ and $A_{3}$ are non-empty subsets of $H$, then
(1) $A \subseteq(A]$;
(2) $\left(A_{1} \cup A_{2}\right]=\left(A_{1}\right] \cup\left(A_{2}\right]$;
(3) $\left(f\left(\left(A_{1}\right],\left(A_{2}\right],\left(A_{2}\right]\right)\right]=\left(f\left(A_{1}, A_{2}, A_{3}\right)\right]$. 
The following lemma is easy to see:

Lemma 2.8. Let $x$ be an element of an ordered ternary semihypergroup $(H, f, \leq)$.

(1) $(x \cup f(H, H, x)]$ is a left hyperideal of $H$ containing $x$.

(2) $(x \cup f(x, H, H)]$ is a right hyperideal of $H$ containing $x$.

(3) $(x \cup f(H, x, H) \cup f(H, f(H, x, H), H)]$ is a middle hyperideal of $H$ containing $x$.

(4) $(x \cup f(H, H, x) \cup f(x, H, H) \cup f(H, f(H, x, H), H)]$ is a two-sided hyperideal of $H$ containing $x$.

(5) $(x \cup f(H, H, x) \cup f(x, H, H) \cup f(H, x, H) \cup f(H, f(H, x, H), H)]$ is a hyperideal of $H$ containing $x$.

\section{BI-HYPERIDEALS}

In this section, we define the notion of bi-hyperideals of an ordered ternary semihypergroup. We introduce the notions of left and right simple ordered ternary semihypergroups and we prove that an ordered ternary semihypergroup is left and right simple if and only if it does not contain proper bi-hyperideals.

Definition 3.1. A ternary subsemihypergroup $A$ of an ordered ternary semihypergroup $(H, f, \leq)$ is called a bi-hyperideal of $H$ if it satisfies the following conditions:

(i) $f(f(A, H, A), H, A) \subseteq A$;

(ii) if $x \in A$ and $y \in H$ such that $y \leq x$, then $y \in A$.

Example 3.2. Suppose that $H=[0,1]$, the unit real interval numbers. We define a ternary hyperoperation $f$ on $H$ as follows:

$$
f(x, y, z)=[0, x y z],
$$

and suppose that $\leq$ is the ordinary order on $[0,1]$. Then, it is easy to see that $(H, f, \leq)$ is an ordered ternary semihypergroup. Now, let $A=[0, a]$ for some a in $A$. Then for every $x, y, z$ in $A$ and $u, v$ in $H$, we have

$$
\begin{aligned}
f(f(x, u, y), v, z) & =f([0, x u y], v, z) \\
& =\bigcup_{t \leq x u y}[0, t v z] \\
& =[0, \text { xuyvz }] .
\end{aligned}
$$

Since $x \leq a, y \leq a$ and $z \leq a$ we obtain xuyvz $\leq a$. Thus $[0, x u y v z] \subseteq A$. Therefore, the first condition of the definition is valid. The second condition of the definition is straighforward. Therefore, $A$ is a bi-hyperideal of $H$.

Definition 3.3. An ordered ternary semihypergroup $(H, f, \leq)$ is said to be left (respectively, middle, right) simple if it does not contain proper left (respectively, middle, right) hyperideals.

Lemma 3.4. An ordered ternary semihypergroup $(H, f, \leq)$ is left simple if and only if $(f(H, H, x)]=H$ for all $x$ in $H$. 
Proof. Assume that $(f(H, H, x)]=H$ for all $x$ in $H$. Let $A$ be a left hyperideal of $H$, and let $a \in A$. Then $(f(H, H, a)]=H$. If $y \in H$, then $y \in(f(H, H, a)]$; hence $y \in f(z, w, a)$ for some $z, w$ in $H$. Since $f(z, w, a) \subseteq A$, we have $y \in A$, and so $A=H$. The opposite direction follows by $(f(H, H, x)]$ is a left hyperideal of $H$ for all $x$ in $H$.

Similarly we have the following lemmas:

Lemma 3.5. An ordered ternary semihypergroup $(H, f, \leq)$ is right simple if and only if $(f(x, H, H)]=H$ for all $x$ in $H$.

Lemma 3.6. An ordered ternary semihypergroup $(H, f, \leq)$ is middle simple if and only if $(f(H, x, H)]=H$ for all $x$ in $H$.

Definition 3.7. An ordered ternary semihypergroup $(H, f, \leq)$ is said to be regular if for any $a \in H, a \in(f(a, H, a)]$.

Lemma 3.8. If an ordered ternary semihypergroup $(H, f, \leq)$ is left and right simple, then it is regular.

Proof. Assume that $H$ is left and right simple. If $a \in H$, then by Lemmas 3.4 and 3.5 we have $H=(f(H, H, a)]$ and $H=(f(a, H, H)]$. Hence

$$
a \in H=(f(a, H, H)]=\left(\begin{array}{c}
f(a, H, f(H, H, a))] \\
(f(a, H, a)] .
\end{array}\right.
$$

Now, we prove the main result of this section:

Theorem 3.9. Let $(H, f, \leq)$ be an ordered ternary semihypergroup. Then $(H, f, \leq)$ is left and right simple if and only if it does not contain proper bi-hyperideals.

Proof. Assume that $(H, f, \leq)$ is left and right simple. Let $A$ be a bi-hyperideal of $H$. We will show that $H \subseteq A$. Let $x \in H$ and $y \in A$. Since $H$ is left simple, we have $H=(y \cup f(H, H, y)]$. Then $x \leq y$ or $x \in(f(z, w, y)]$ for some $z, w$ in $H$. If $x \leq y$, then $x \in A$. Assume that $x \in(f(z, w, y)]$. Since $H$ is right simple, we have $H=(y \cup f(y, H, H)]$. Since $z \in H$, so $z \leq y$ or $z \in(f(y, u, v)]$ for some $u, v$ in $H$. By Lemma 3.8, $(H, f, \leq)$ is regular. Then there exists $b \in H$ such that $y \in(f(y, b, y)]$.

If $z \leq y$, then

$$
\begin{aligned}
(f(y, w, y)] \subseteq(f((f(y, b, y)], w, y)] & \subseteq(f((f(A, H, A)], H, A)] \\
& \subseteq(f(f(A, H, A), H, A)] \\
& \subseteq A
\end{aligned}
$$

hence $x \in A$.

$$
\text { If } z \in f(y, u, v) \text {, then }
$$

$(f((f(y, u, v)], w, y)] \subseteq(f((f(y, u, v)], w,(f(y, b, y)])] \subseteq(f(f(A, H, A), H, A)] \subseteq A ;$

hence $x \in A$. Thus $H \subseteq A$. 
Conversely, assume that $(H, f, \leq)$ does not contain proper bi-hyperideals. If $A$ is a left hyperideal of $H$, then $A$ is a bi-hyperideal of $H$. By assumption, $H=A$. Similarly, if $A$ is a right hyperideal of $H$, then $A$ is a bi-hyperideal of $H$, so $H=A$.

\section{QUASI-HYPERIDEALS}

In this section we define the notion of quasi-hyperideals of an ordered ternary semihypergroup and we study some of their properties.

Definition 4.1. A non-empty subset $Q$ of an ordered ternary semihypergroup $(H, f, \leq)$ is called a quasi-hyperideal of $H$ if it satisfies the following conditions:

(i) $f(H, H, Q) \cap f(H, Q, H) \cap f(Q, H, H) \subseteq Q$;

(ii) $f(H, H, Q) \cap f(H, f(H, Q, H), H) \cap f(Q, H, H) \subseteq Q$;

(iii) if $x \in Q$ and $y \in H$ such that $y \leq x$, then $y \in Q$.

Example 4.2. All left hyperideals $A, B$ and $C$ defined in Example 2.7 are quasihyperideals.

Note that every quasi-hyperideal is a ternary subsemihypergroup.

Theorem 4.3. Let $Q$ be a quasi-hyperideal of an ordered ternary semihypergroup $(H, f, \leq)$. If $T$ is a ternary subsemihypergroup of $H$, then $Q \cap T=\emptyset$ or $Q \cap T$ is a quasi-hyperideal of $T$.

Proof. Let $Q_{1}=Q \cap T$. Assume that $Q_{1} \neq \emptyset$. Since $Q_{1} \subseteq Q$, it follows that $f\left(T, T, Q_{1}\right) \cap f\left(T, Q_{1}, T\right) \cap f\left(Q_{1}, T, T\right) \subseteq f(H, H, Q) \cap f(H, Q, H) \cap f(Q, H, H) \subseteq Q$. Since $Q_{1} \subseteq T$ and $T$ is a ternary subsemihypergroup of $H$, we have

$$
f\left(T, T, Q_{1}\right) \cap f\left(T, Q_{1}, T\right) \cap f\left(Q_{1}, T, T\right) \subseteq T .
$$

Then

$$
f\left(T, T, Q_{1}\right) \cap f\left(T, Q_{1}, T\right) \cap f\left(Q_{1}, T, T\right) \subseteq Q_{1} .
$$

If $x \in Q_{1}$ and $y \in T$ such that $y \leq x$, then since $x \in Q$ we have $y \in Q$; hence $y \in Q_{1}$. Therefore, $Q_{1}$ is a quasi-hyperideal of $T$.

Theorem 4.4. Let $\left\{Q_{i} \mid i \in I\right\}$ be an indexed family of quasi-hyperideals of an ordered ternary semihypergroup $(H, f, \leq)$. If $\bigcap_{i \in I} Q_{i} \neq \emptyset$, then it is a quasi-hyperideal of $H$.

Proof. Let $Q=\bigcap_{i \in I} Q_{i}$. Since, for each $i \in I$,

$$
\begin{gathered}
f(H, H, Q) \cap f(H, Q, H) \cap f(Q, H, H) \subseteq \\
f\left(H, H, Q_{i}\right) \cap f\left(H, Q_{i}, H\right) \cap f\left(Q_{i}, H, H\right) \subseteq Q_{i},
\end{gathered}
$$

we have

$$
f(H, H, Q) \cap f(H, Q, H) \cap f(Q, H, H) \subseteq Q .
$$

Similarly, we have

$$
f(H, H, Q) \cap f(H, f(H, Q, H), H) \cap f(Q, H, H) \subseteq Q .
$$


If $x \in Q$ and $y \in H$ such that $y \leq x$, then for each $i \in I$ we have $y \in Q_{i}$; hence $y \in Q$. Therefore, $Q$ is a quasi-hyperideal of $H$.

Let $A$ be a non-empty subset of an ordered ternary semihypergroup $(H, f, \leq)$. Then the intersection of all quasi-hyperideals of $H$ containing $A$, denoted by $(A)_{q}$, is a quasi-hyperideal of $H$ containing $A$. This is called the quasi-hyperideal of $H$ generated by $A$.

Theorem 4.5. The intersection of a left, a middle and a right hyperideals of an ordered ternary semihypergroup $(H, f, \leq)$ is a quasi-hyperideal of $H$.

Proof. Let $L, M$ and $R$ be a left hyperideal, a middle hyperideal and a right hyperideal of $H$, respectively. Let $Q=L \cap M \cap R$. We choose $l \in L, m \in M$ and $r \in R$. Since $f(l, m, r) \subseteq L \cap M \cap R$, so $Q \neq \emptyset$. Since

$$
f(H, H, Q) \subseteq L, f(H, Q, H) \subseteq M \text { and } f(Q, H, H) \subseteq R,
$$

it follows that

$$
f(H, H, Q) \cap f(H, Q, H) \cap f(Q, H, H) \subseteq L \cap M \cap R=Q .
$$

Similarly,

$$
f(H, H, Q) \cap f(H, f(H, Q, H), H) \cap f(Q, H, H) \subseteq Q .
$$

If $x \in Q$ and $y \in H$ such that $y \leq x$, then by $x \in L \cap M \cap R$ we have $y \in L \cap M \cap R$. Hence $Q$ is a quasi-hyperideal of $H$.

Theorem 4.6. If $Q$ is a quasi-hyperideal of an ordered ternary semihypergroup $(H, f, \leq)$, then there exist a left hyperideal $L$, a middle hyperideal $M$ and a right hyperideal $R$ of $H$ such that $Q=L \cap M \cap R$.

Proof. Assume that $Q$ is a quasi-hyperideal of $H$. Let

$$
\begin{aligned}
L & =(Q \cup f(Q, H, H)] \\
R & =(Q \cup f(H, H, Q)] \\
M & =(Q \cup f(H, Q, H) \cup f(H, f(H, Q, H), H)] .
\end{aligned}
$$

We have $L \neq \emptyset$. Let $x \in f(L, H, H)$, then $x \in f\left(l, h, h^{\prime}\right)$ for some $l$ in $L$ and $h, h^{\prime}$ in $H$. Let $l \leq p$ for some $p$ in $Q \cup f(Q, H, H)$. Then $f\left(l, h, h^{\prime}\right) \leq f\left(p, h, h^{\prime}\right)$. There are two cases to consider:

Case 1: $p \in Q$. By

$$
f\left(p, h, h^{\prime}\right) \subseteq f(Q, H, H) \subseteq Q \cup f(Q, H, H),
$$

it follows $x \in L$. Since

Case 2: $p \in f(Q, H, H)$. Let $p \in f\left(q, h_{1}, h_{2}\right)$ for some $q$ in $Q$ and $h_{1}, h_{2}$ in $H$.

$$
\begin{gathered}
f\left(p, h, h^{\prime}\right) \subseteq f\left(f\left(q, h_{1}, h_{2}\right) h, h^{\prime}\right) \subseteq f\left(q, f\left(h_{1}, h_{2}, h\right), h^{\prime}\right) \subseteq f(Q, H, H) \subseteq \\
Q \cup f(Q, H, H),
\end{gathered}
$$

we have $x \in L$. Then $f(L, H, H) \subseteq L$. Let $x \in L$ and $y \in H$ be such that $y \leq x$. Then $x \leq z$ for some $z$ in $Q \cup f(Q, H, H)$. Since $y \leq z, y \in L$. Hence $L$ is a left 
hyperideal of $H$. Similarly, $R$ is a right hyperideal of $H$. To show that $M$ is a middle hyperideal of $H$, let $x \in f(H, M, H]$. Then $x \in f\left(h_{3}, m, h_{4}\right)$ for some $m$ in $M$ and $h_{3}, h_{s}$ in $H$. Let $m \leq n$ for some $n$ in $Q \cup f(H, Q, H) \cup f(H, f(H, Q, H), H)$. Then $f\left(h_{3}, m, h_{4}\right) \subseteq f\left(h_{3}, n, h_{4}\right)$. There are three cases to consider:

Case 1: $n \in Q$. Since $f\left(h_{3}, n, h_{4}\right) \subseteq f(H, Q, H)$, so $x \in M$.

Case 2: $n \in f(H, Q, H)$. Since $f\left(h_{3}, n, h_{4}\right) \subseteq f(H, f(H, Q, H), H)$, so $x \in M$.

Case 3: $n \in f(H, f(H, Q, H), H)$. Since $f\left(h_{3}, n, h_{4}\right) \subseteq f(H, Q, H)$, so $x \in M$. Therefore, $f(H, M, H) \subseteq M$.

Let $x \in M$ and $y \in H$ be such that $y \leq x$. Then $x \leq z$ for some $z \in$ $Q \cup f(H, M, H) \cup f(H, f(H, Q, H), H)$. Thus $y \in M$.

We will show that $Q=L \cap M \cap R$. It is clear that $Q \subseteq L \cap M \cap R$. Since $Q$ is a quasi-hyperideal of $H$, we have that

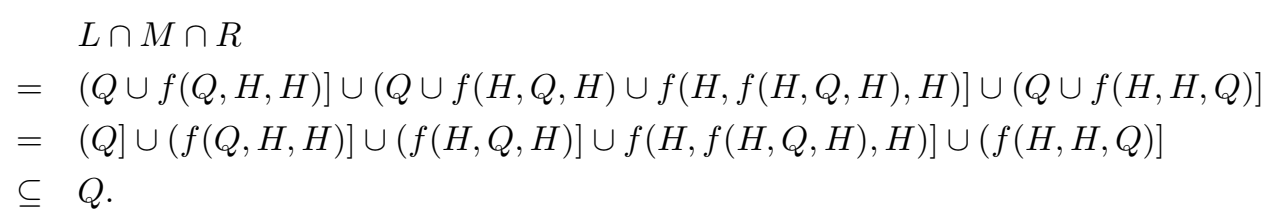

This completes the proof.

Theorem 4.7. Let $A$ be a non-empty subset of an ordered ternary semihypergroup $(H, f, \leq)$. Then the intersection of all quasi-hyperideals of $H$ containing $A$ is of the form

$(A)_{q}=(A \cup f(H, H, A)] \cap(A \cup f(H, A, H) \cup f(H, f(H, A, H), H)] \cap(A \cup f(A, H, H)]$.

Proof. Let $Q$ be the intersection of all quasi-hyperideals $Q_{i}, i \in I$ of $H$ containing $A$. By Theorem 4.4, $(A)_{q}$ is a quasi-hyperideal of $H$ containing $A$. We have

$(A \cup f(H, H, A)],(A \cup f(H, A, H) \cup f(H, f(H, A, H), H)]$ and $(A \cup f(A, H, H)]$

are left, middle and right hyperideals of $H$, respectively. By Theorem 4.5,

$(A \cup f(H, H, A)] \cap(A \cup f(H, A, H) \cup f(H, f(H, A, H), H)] \cap(A \cup f(A, H, H)]$

is a quasi-hyperideal of $H$ containing $A$. Thus

$(A)_{q} \subseteq(A \cup f(H, H, A)] \cap(A \cup f(H, A, H) \cup f(H, f(H, A, H), H)] \cap(A \cup f(A, H, H)]$.

For each $i \in I$, since $A \subseteq Q_{i}$, we have

$$
\begin{aligned}
& (A \cup f(H, H, A)] \cap(A \cup f(H, A, H) \cup f(H, f(H, A, H), H)] \cap(A \cup f(A, H, H)] \\
= & (A] \cup(f(H, H, A)] \cap(f(H, A, H) \cup f(H, f(H, A, H), H)] \cap(f(A, H, H)] \\
\subseteq & \left(Q_{i}\right] \cup\left(f\left(H, H, Q_{i}\right)\right] \cap\left(f\left(H, Q_{i}, H\right) \cup f\left(H, f\left(H, Q_{i}, H\right), H\right)\right] \cap\left(f\left(Q_{i}, H, H\right)\right] \\
\subseteq & Q_{i} .
\end{aligned}
$$

Then

$(A)_{q} \supseteq(A \cup f(H, H, A)] \cap(A \cup f(H, A, H) \cup f(H, f(H, A, H), H)] \cap(A \cup f(A, H, H)]$.

We have the assertion. 
Definition 4.8. An ordered ternary semihypergroup $(H, f, \leq)$ is said to be quasisimple if it is the unique quasi-hyperideal of itself.

Theorem 4.9. An ordered ternary semihypergroup $(H, f, \leq)$ is quasi-simple if and only if

$$
H=(f(x, H, H)] \cap(f(H, x, H)] \cup(f(H, f(H, x, H), H)] \cap(f(H, H, x)]
$$

for all $x$ in $H$.

Proof. Assume that $H$ is quasi-simple. Let $x \in H$. We shall show that $L=$ $(f(x, H, H)]$ is a left hyperideal of $H$. Let $y \in f(L, H, H)$. Then $y \in f\left(l, h, h^{\prime}\right)$ for some $l$ in $L$ and $h, h^{\prime}$ in $H$. Since $l \in L, l \in f\left(x, h_{1}, h_{2}\right)$ for some $h_{1}, h_{2}$ in $H$. Thus

$$
y \in f\left(l, h, h^{\prime}\right) \subseteq f\left(f\left(x, h_{1}, h_{2}\right), h, h^{\prime}\right)=f\left(x, f\left(h_{1}, h_{2}, h\right), h^{\prime}\right) \subseteq f(x, H, H) \subseteq L .
$$

Let $y \in L$ and $z \in H$ be such that $z \leq y$. Since $y \in L, y \leq w$ for some $w$ in $f(x, H, H)$. Since $z \leq w, z \in(f(x, H, H)]=L$. Therefore, $L$ is a left hyperideal of $H$. Similarly, $f(H, H, x)$ is a right hyperideal of $H$. To show that $M=f(H, x, H) \cup$ $f(H, f(H, x, H), H)$ is a middle hyperideal of $H$, let $y \in f(H, M, H)$. Then $y \in$ $f\left(h, m, h^{\prime}\right)$ for some $m$ in $M$ and $h, h^{\prime}$ in $H$. If $m \in f\left(h_{1}, x, h_{2}\right)$ for some $h_{1}, h_{2}$ in $H$, then

$$
y \in f\left(h, m, h^{\prime}\right) \subseteq f\left(h, f\left(h_{1}, x, h_{2}\right), h^{\prime}\right) \subseteq f(H, f(H, x, H), H) \subseteq M .
$$

If $m \in f\left(h_{3}, f\left(h_{4}, x, h_{5}\right), h_{6}\right)$ for some $h_{3}, h_{4}, h_{5}, h_{6}$ in $H$, then

$$
y \in f\left(h, m, h^{\prime}\right) \subseteq f\left(h, f\left(h_{3}, f\left(h_{4}, x, h_{5}\right), h_{6}\right), h^{\prime}\right) \subseteq f(H, x, H) \subseteq M .
$$

Then $f(H, M, H) \subseteq M$. Let $y \in M$ and $z \in H$ be such that $z \leq y$. If $y \leq w$ for some $w$ in $f(H, f(H, x, H), H)$, then $z \in(f(H, f(H, x, H), H)] \subseteq M$. If $y \leq w^{\prime}$ for some $w^{\prime}$ in $f(H, x, H)$, then $z \in(f(H, x, H)] \subseteq M$. Therefore, $M$ is a middle hyperideal of $H$. By Theorem 4.4,

$$
H=(f(x, H, H)] \cap(f(H, x, H)] \cup(f(H, f(H, x, H), H)] \cap(f(H, H, x)] .
$$

Conversely, assume that

$$
H=(f(x, H, H)] \cap(f(H, x, H)] \cup(f(H, f(H, x, H), H)] \cap(f(H, H, x)]
$$

for all $x$ in $H$. Let $Q$ be a quasi-hyperideal of $H$. If $q \in Q$, then by assumption we get

$$
\begin{aligned}
H & =(f(q, H, H)] \cap(f(H, q, H)] \cup(f(H, f(H, q, H), H)] \cap(f(H, H, q)] \\
& \subseteq(f(Q, H, H)] \cap(f(H, Q, H)] \cup(f(H, f(H, Q, H), H)] \cap(f(H, H, Q)] \\
& \subseteq Q .
\end{aligned}
$$

Thus $H=Q$. This completes the proof.

Definition 4.10. A quasi-hyperideal $Q$ of an ordered ternary semihypergroup $(H, f, \leq)$ is said to be minimal if it contains no proper quasi-hyperideal of $H$.

Theorem 4.11. Every quasi-simple hyperideal of an ordered ternary semihypergroup $(H, f, \leq)$ is minimal. 
Proof. Let $Q$ be a quasi-simple hyperideal of $H$. Let $Q^{\prime}$ be a quasi-hyperideal of $H$ such that $Q^{\prime} \subseteq Q$. Since $Q^{\prime}$ is a quasi-hyperideal of $Q, Q^{\prime}=Q$. Then $Q$ is a minimal quasi-hyperideal of $H$.

\section{REFERENCES}

[1] Anvariyeh, S.M., Mirvakili, S. and Davvaz, B., Combinatorial aspects of $n$-ary polygroups and $n$-ary color schemes, European Journal of Combinatorics, 34 (2013), 207-216.

[2] Bonansinga, P. and Corsini, P., On semihypergroup and hypergroup homomorphisms, Boll. Un. Mat. Ital. B (6) 1(2) (1982), 717-727.

[3] Chvalina, J., Commutative hypergroups in the sense of Marty and ordered sets, General algebra and ordered sets (Horn Lipova, 1994), 19-30.

[4] Davvaz, B., Some results on congruences in semihypergroups, Bull. Malays. Math. Soc. (2), 23 (2000), 53-58.

[5] Davvaz, B., Characterizations of sub-semihypergroups by various triangular norms, Czechoslovak Mathematical Journal, 55(4) (2005), 923-932.

[6] Davvaz, B. and Leoreanu-Fotea, V., Binary relations on ternary semihypergroups, Communications in Algebra, 38(10) (2010) 3621-3636.

[7] Davvaz, B. and Vougiouklis, T., n-Ary hypergroups, Iran. J. Sci. Technol. Trans. A, 30(2) (2006), 165-174.

[8] De Salvo, M., Freni, D. and Lo Faro, G., Fully simple semihypergroups, J. Algebra, 399 (2014), 358-377.

[9] Dörnte, W., Untersuchungen ber einen verallgemeinerten Gruppenbegriff, Mathematische Zeitschrift, 29(1) (1929) 1-19.

[10] Dudek, W.A., On divisibility in n-semigroups, Demonstratio Math., 13 (1980), 355-367.

[11] Freni, D., Minimal order semihypergroups of type $U$ on the right, II, J. Algebra, 340 (2011), 77-89.

[12] Heidari, D. and Davvaz, B., On ordered hyperstructures, Politehn. Univ. Bucharest Sci. Bull. Ser. A Appl. Math. Phys., 73(2) (2011), 85-96.

[13] Hila, K., Naka, K., Leoreanu-Fotea, V. and Sadiku, S., Algebraic hyperstructures of soft sets associated with ternary semihypergroups, Ital. J. Pure Appl. Math., 30 (2013), 349-372.

[14] Hila, K., Davvaz, B. and Naka, K., On Quasi-hyperideals in semihypergroups, Communications in Algebra, 39 (2011), 4183-4194.

[15] Hila, K., Davvaz, B. and Dine, J., Study on the structure of $\Gamma$-semihypergroups, Communications in Algebra, 40(8) (2012), 2932-2948.

[16] Iampan, A., On ordered ideal extensions of ordered ternary semigroups, Lobachevskii J. Math., 31(1) (2010), 13-17.

[17] Marty, F., Sur une generalization de la notion de groupe, $8^{i e m}$ Congres Math. Scandinaves, Stockholm, (1934) 45-49.

[18] Mirvakili, S. and Davvaz, B., Applications of strongly transitive geometric spaces to $n$-ary hypergroups, ARS Combinatoria, 109 (2013), 193-227.

[19] Mirvakili, S. and Davvaz, B., On some combinatorial aspects of transposition $n$-ary hypergroups, Carpathian Journal of Mathematic, 30(1) (2014), 109-116.

[20] Naka, K. and Hila, K., Some properties of hyperideals in ternary semihypergroups, Math. Slovaca, 63(3) (2013), 449-468.

[21] Lehmer, D.H., A ternary analogue of abelian groups, American Journal of Mathematics, 54 (1932), 329-338.

[22] Leoreanu, V., About the simplifiable cyclic semihypergroups, Ital. J. Pure Appl. Math., 7 (2000), 69-76.

[23] Leoreanu-Fotea, V. and Davvaz, B., n-hypergroups and binary relations, European Journal of Combinatorics, 29 1207-1218. 
[24] Los, J., On the extending of models I, Fundamenta Mathematicae, 42 (1955), 38-54.

[25] M.L. Santiago, M.L. and Bala, S.S., Ternary semigroups, Semigroup Forum, 81 (2010) 380-388.

[26] Sioson, F.M., Ideal theory in ternary semigroups, Mathematica Japonica, 10 (1965) 63-84. 